\title{
Effect of Spencer Muscle Energy Technique Versus Maitland's Mobilization Technique on Pain, ROM and Disability in Patients with Frozen Shoulder: A Comparative Study
}

\author{
Raksha R. Jivani ${ }^{{ }^{*}}$, Dharti N Hingarajia ${ }^{2}$. \\ ${ }^{* 1}$ Assistant Physiotherapist, sahyog physiotherapy and fitness centre, Surat, Gujarat, India. \\ ${ }^{2}$ Sr. Lecturer, SPB Physiotherapy College, Surat, Gujarat, India.
}

\section{ABSTRACT}

Background: Adhesive capsulitis is characterized by a painful, gradual loss of both active and passive glenohumeral motion resulting from progressive fibrosis and ultimate contracture of the glenohumeral joint capsule. Patients with Adhesive capsulitis have difficulties in everyday activities and shoulder pain also disturbs sleep at night on the affected side. Muscle energy technique helps in increasing shoulder range of motion. Maitland Mobilization is commonly used in the treatment of frozen shoulder. SPENCER Muscle Energy Technique (MET) is unique in its application as the client provides the initial effort while the practitioner facilitates the process.

Objective: Objectives of the study was to compare the effect of Spencer MET Vs Maitland's mobilization on pain, Range of Motion (ROM) and Disability in the patients with frozen shoulder.

Methods: In the present experimental study, total 58 patients with frozen shoulder were included. Inclusion criteria were male and female with age of 40 to 60 year with unilateral frozen shoulder (at least 3-month duration). Patients were randomly allocated in two groups with 29 patients in each group: SPENCER MET and Conventional physiotherapy and $\mathrm{MM}$ and conventional physiotherapy for 5 days a week with total duration of 4 weeks. Pre and post intervention assessment was carried out by using VAS, SPADI and ROM. Data was analysed by using SPSS 15 version.

Results: Paired t test was applied within group comparison and result showed statistically significant difference in post intervention measurement compared to pre intervention for improving pain, reducing disability and increasing all ROM in both the groups. Independent t test was applied between group comparison and result showed statistically significant difference between groups mean pre-post differences in improving pain, reducing disability and increasing all ROM except extension and internal rotation.

Conclusion: This study concludes that both the techniques used in the present study i.e., Spencer Muscle Energy Technique and Maitland Mobilization are effective for improving pain, reducing disability, and increasing ROM. However, SPENCER MET is the more effective for improving pain, reducing disability, and increasing ROM compared to Maitland Mobilization in patients with frozen shoulder.

KEY WORDS: Frozen shoulder, SPENCER MET, Maitland mobilization, Shoulder Pain and Disability Index, Visual Analogue Scale.

Address for correspondence: Raksha R. Jivani, Assistant Physiotherapist, Sahyog Physiotherapy and Fitness Centre, Surat, Gujarat, India. E-mail: rakshajivani4163@gmail.com

Access this Article online

Quick Response code

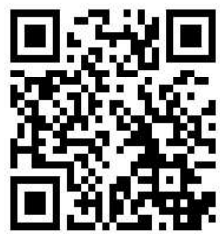

DOI: $10.16965 /$ ijpr.2021.148

\section{Journal Information}

\section{International Journal of Physiotherapy and Research}

ISSN (E) 2321-1822 | ISSN (P) 2321-8975

https://www.ijmhr.org/ijpr.html

DOI-Prefix: https://dx.doi.org/10.16965/ijpr

\section{Article Information}

Received: 28 May 2021

Peer Review: 29 May 2021

Revised: None
Accepted: 13 Jul 2021

Published (O): 11 Aug 2021

Published (P): 11 Aug 2021 


\section{INTRODUCTION}

Frozen shoulder is a painful shoulder condition of insidious onset that was associated with stiffness and difficulty in sleeping on the affected side. It is characterized by a painful, gradual loss of both active and passive glenohumeral motion resulting from progressive fibrosis and ultimate contracture of the glenohumeral joint capsule and the marked reduction in forward elevation and external rotation i.e., Capsular pattern of involvement $[1,2]$.

The incidence of FS is slightly higher in women than in men (70\% of patients are women). This condition most frequently affects persons aged 40 to 60 years and rarely occurs in persons younger than 40 years of age. Frozen shoulder might affect both shoulders in up to $16 \%$ of patients; however, a relapse is uncommon. Prevalence rate in the general population is $2-5 \%$ and $10-20 \%$ in diabetics [3]. An increased incidence of frozen shoulder has been noticed in patients with hyperthyroidism and hypertriglyceridemia [4].

The restriction of the shoulder movement is thought to be the result of inflammation and swelling in the lining of the shoulder joint capsule and its associated ligaments with resultant contracture of the shoulder joint capsule. The lining loses its normal characteristic of the flexibility and becomes stiff and painful [5]. In frozen shoulder joint capsule get thickens, swells, and tightens due to bands of scar tissue (adhesion) that have formed inside the capsule [6].

Frozen shoulder progressive through three clinical phases (1) painful phase- severe pain usually worst at night and when lying on the affected side (2-9 months) (2) are stiffening or frozen phase- difficulty with simple activities of daily living. Stiffness progresses and leads to disused atrophy (4-12 months) (3) Thawing phase - gradual increase in range of motion and improvement in pain, although it may reappear as stiffness ceases (5-12 months) [7].

The traditional principles of treatment of adhesive capsulitis are pain relief, maintain range of motion, and ultimately to restore function. Treatments advocated for frozen shoulder include physiotherapy interventions such as hot therapy, electro modalities and exercise programs consist of active and passive ROM exercises, stretching exercises guided by a physiotherapist, self-stretching, manipulation and mobilization techniques, strengthening exercises, patient education and home exercises.

Muscle energy technique (MET) is unique in its application as the client provides the initial effort while the practitioner facilitates the process. One of the main uses of this method is to normalize joint range, rather than increase flexibility, and techniques can be used on any joints with restricted Range of Motion (ROM) identified during the passive assessment. The main effects of MET can be explained by two distinct physiological processes: Post Isometric Relaxation (PIR) and Reciprocal Inhibition (RI) [8].

Spencer MET is one of the special techniques of MET. It is unique in its application as the client provides the initial effort while the practitioner facilitates the process. It is a standardized series of shoulder treatments with broad application in diagnosis, treatment, and prognosis. It is developed by Spencer, D.O. in 1916. The evolution of this technique is traced form 1916 to date to try to identify factors in the development of manipulative methods [9]. Spencer technique is an articulatory technique with seven different procedures used to treat shoulder restriction caused by adhesive capsulitis. In this technique passive, smooth, rhythmic motion is designed to stretch contracted muscles, ligaments, and capsules. Most of the force is applied at the end range of motion. This technique increases pain free range of motion through stretching the tissues, enhancing lymphatic flow, and stimulating increased joint circulation [10]. Few studies in literature found the effectiveness of Spencer Muscle Energy Technique in frozen shoulder [11].

Maitland mobilization (MM) is a manual technique commonly used in the physiotherapy management of FS [12]. It applies passive oscillations, classified from Grade I-IV with respect to amplitude and intensity, to the shoulder in order to treat pain and stiffness. 
Grades I and II of Maitland mobilization techniques are primarily used for treating joints limited by pain. Whereas Grades III and IV are primarily used as stretching manoeuvre [13]. There are few previous studies which prove the effect of MM on pain and ROM among FS patients [12].

Various studies prove the effect of Spencer MET and MM on pain, ROM and Disability in patients with frozen shoulder individually. However, there are very few studies found in the literature which compare the effects of Spencer MET and Maitland's mobilization technique.

Therefore, there was a need to find the comparative effect of two treatment techniques for subjects with frozen shoulder. Hence the purpose of the present study was to compare the effect of spencer muscle energy technique Versus Maitland's manual mobilization technique on pain, ROM, and disability in patients with frozen shoulder.

\section{AIMS AND OBJECTIVES}

\section{Primary objective:}

1. To compare the effect of Spencer MET Vs Maitland's mobilization on pain, ROM and Disability in the patients with frozen shoulder.

\section{Secondary objectives:}

2. To evaluate the effect of Spencer MET before and after intervention on pain, ROM and disability in patients with frozen shoulder.

3. To evaluate the effect of Maitland's mobilization before and after intervention on pain, ROM and disability in patients with frozen shoulder.

\section{HYPOTHESIS:}

Null hypothesis $\left(\mathbf{H}_{\mathbf{0}}\right)$ : There will be no significant difference between the effect of Spencer MET and Maitland's Mobilisation on pain, ROM and disability in patients with frozen shoulder.

Alternative hypothesis $\left(\mathbf{H}_{1}\right)$ : There will be significant difference between the effect of Spencer MET and Maitland's Mobilisation on pain, ROM and disability in patients with frozen shoulder.

\section{METHODOLOGY}

Study Design: Pre and post-test experimental study.

Population: Patients with Frozen shoulder.

Sampling Method: Purposive sampling

\section{Study Duration: 1 year}

Sample Size: For the study the sample size was calculated in G Power 3.1.9.2 with effect size 0.8 and á $=0.05$ at $80 \%$ power. Sample size calculated was 52 , with a drop out chances of $10 \%$ the total sample size was 58,29 samples in each group.

Study setting: Patients with frozen shoulder were recruited for the study from various physiotherapy OPDs from Surat.

\section{Inclusion Criteria}

1. Subjects with age of 40 to 60 years.

2. Both male and female subjects.

3. Unilateral Frozen shoulder (at least 3-month duration).

\section{Exclusion Criteria}

1. Subjects with recent history of surgery on affected shoulder.

2. Rheumatoid arthritis

3. Subjects with history of any trauma/ fracture around shoulder complex.

4. Osteoporosis and malignancies in shoulder region.

5. Neurological deficits affecting shoulder function

6. Subjects with rotator cuff lesion and, tendon calcification.

7. Pain or disorders of cervical spine, elbow, wrist or hand on affected side

\section{Outcome Measures:}

1. Pain was measured using Visual Analogue Scale (VAS) [14].

2. Mobility i.e., all shoulder range of motions (ROM) were measured using Universal goniometer in degrees $[15,16]$.

3. Functional disability of shoulder was measured using shoulder pain disability index (SPADI) [17].

Procedure: As the study includes human subjects' ethical clearance was obtained from 
ethical committee of SPB Physiotherapy College. The purpose of the study was explained, and a written informed consent was obtained from all the subjects. Subjects were preliminary screened based on the inclusion and exclusion criteria and their demographic data was collected. Pre intervention outcome measures were recorded. Subjects were randomly allocated in to two groups by using closed envelops method. Descriptions of group are as follow:

GROUP A: This consists of 29 patients, and they received conventional exercises and 7 stages of Spencer MET [18]. The total time duration for this treatment was 30 minutes with rest.

GROUP B: This consists of 29 patients, and they received conventional exercises and Maitland's mobilization technique. The total duration of Maitland's mobilization was 20 minutes. Initiation and progression of mobilisation was given according to Maitland approach [19]. 3 glides were given: Caudal glide, Posterior glide, Anterior glide.

Procedure of Blinding: Subjects were blinded on either type of intervention and to which group they were belonged. Throughout the treatment sessions, subjects from both the groups were not allowed to have any interaction to each other and the subjects were not aware of what kind of treatment they received and its effects.

\section{Conventional Therapy for both the groups:}

The subjects were made to do exercises within pain-free range under supervision. The treatment includes were:

1. Modality: hot pack (10 minutes),

2. Exercises:

- Capsular stretching of shoulder joint.

- Isometric strengthening exercise of flexor, extensor, abductors, adductors, external rotators, internal rotators.

- Scapula stabilization exercise: open chain exercises of scapula stabilizers without weight and progression with light weight dumbbells in prone position.

- Active free exercises using cane/wand.

- All the Exercises were performed for all movements namely flexion/extension and abduction-adduction, external and internal rotation.

- One sets of each 10-15 repetitions within pain-free range. (total conventional treatment time is 30 minutes)

Total Treatment Time was for both the groups 1 Hour, 5 Session/Week for 4 Weeks

Statistical Analysis: Statistical analysis was done using SPSS version 15.00 Software. Kolmogrov-smirnov test was applied to check the normality of data. All quantitative data of this study follow the normal distribution $(p>0.05)$. Baseline characteristics were compared by independent t-test to check homogeneity between intervention groups. Paired t-test was used to analyse the pre and post intervention differences within each group and independent t-test was used for between groups comparison. Confidence interval was kept $95 \%$ and the level of significance for all statistical data was set $\alpha=0.05$.

\section{RESULTS AND DISCUSSION}

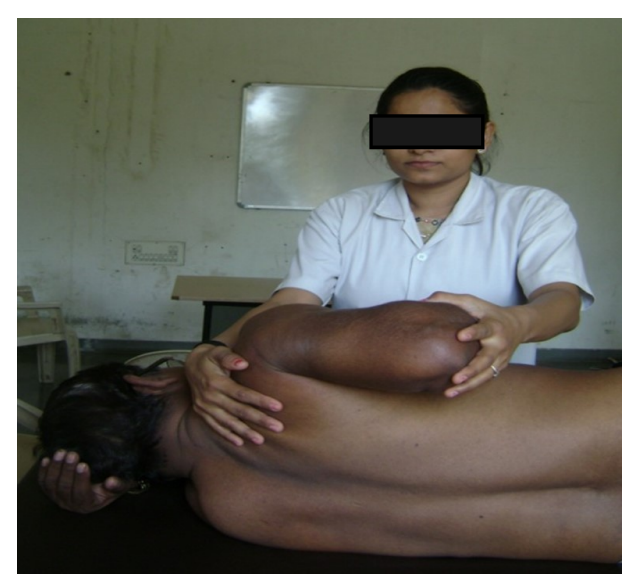

Fig. 1: First stage of SPENCER MET: Shoulder extension.

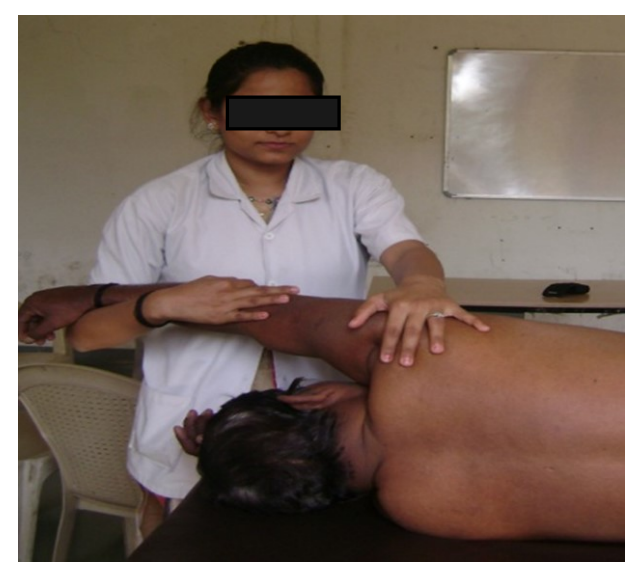

Fig. 2: Second stage of SPENCER MET: Shoulder flexion. 


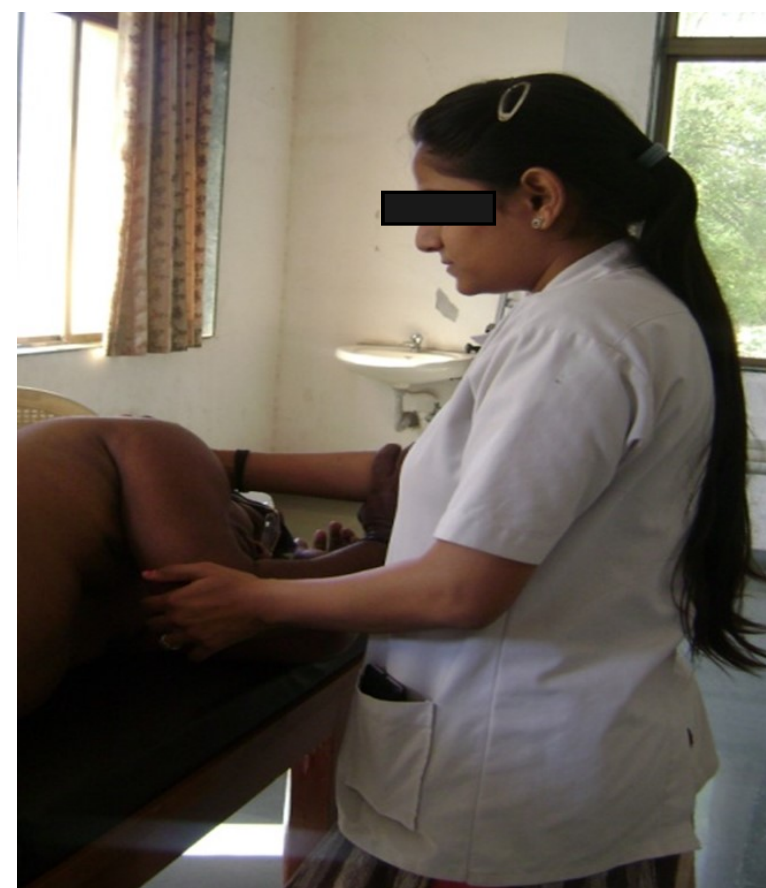

Fig. 3: Third stage of SPENCER MET: Adduction with Internal rotation.

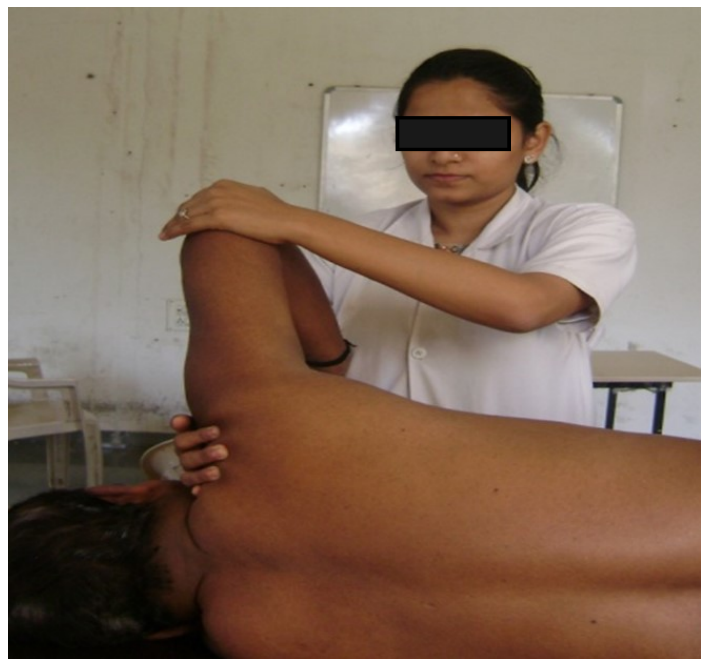

Fig. 4: Fourth stage of SPENCER MET: Abduction with External rotation.

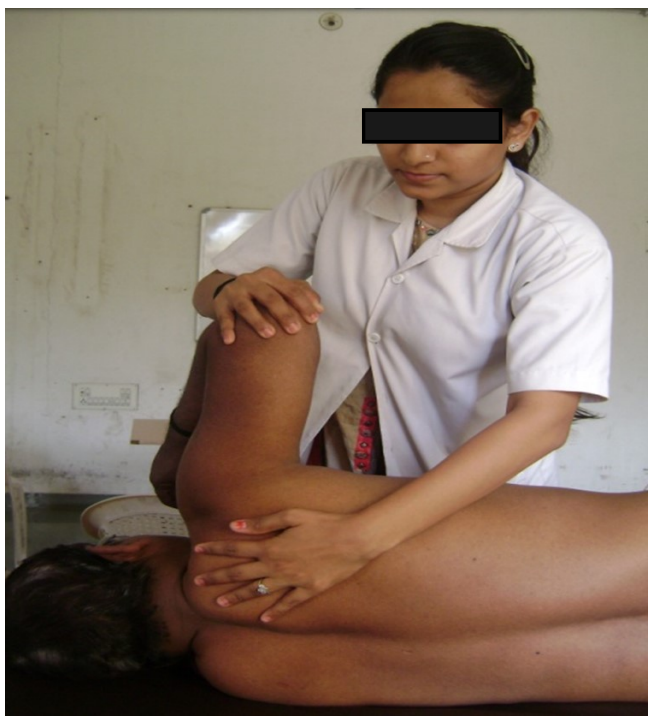

Fig. 5: Fifth stage of SPENCER MET: Compression with circumduction.

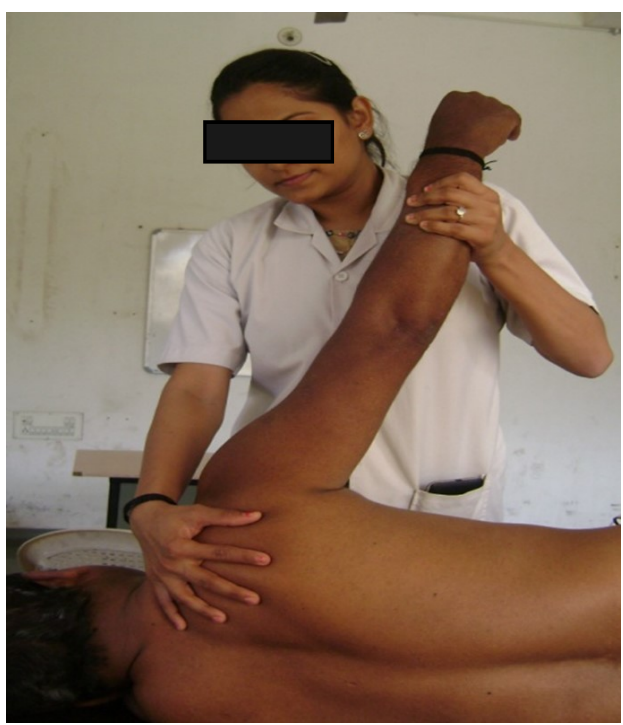

Fig. 6: Sixth stage of SPENCER MET: Distraction with circumduction.

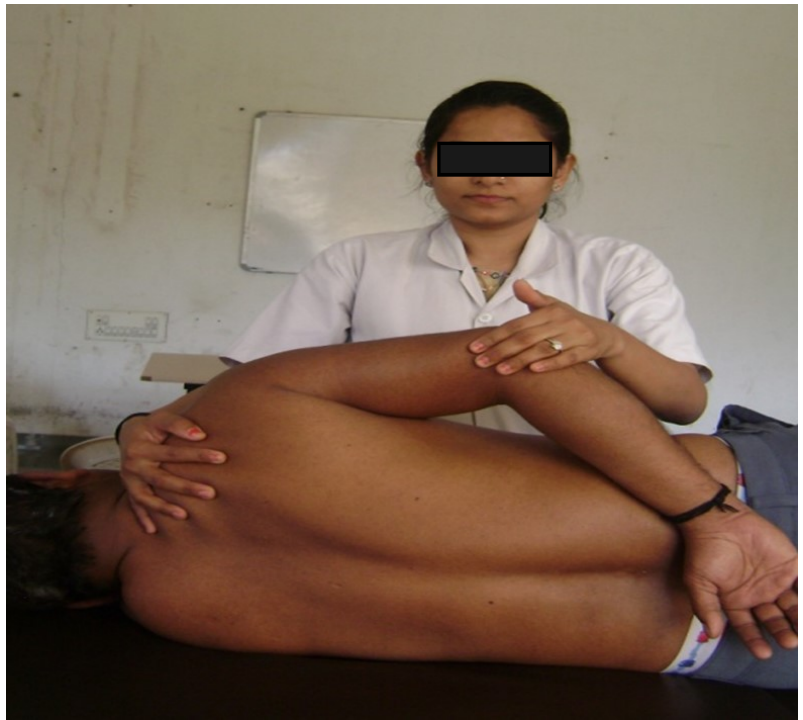

Fig. 7: Seventh stage of SPENCER MET: Shoulder internal rotation

Total 74 patients were assessed for eligibility. Sixteen patients were excluded because they did not meet inclusion Criteria. Fifty-eight patients were enrolled in the study and randomized to one of the treatment groups (29 in SPENCER MET group and 29 in MM group). 8 patients from SPENCER MET and 10 patients from MM group were taking pain reducing medication when assessed before treatment. However, 7 patients from SPENCER MET and 8 patients from MM group discontinued the medicine during intervention period. 2 patients from SPENCER MET and 3 from $\mathrm{MM}$ group discontinued intervention in between. Outcome measurements were completed on 52 participants (27 in the SPENCER MET group and 26 in the MM group) after 4 weeks of intervention. 

ROM and Disability in Patients with Frozen Shoulder: A Comparative Study.

\begin{tabular}{lccc}
\hline \multirow{2}{*}{ Variable } & Group A & Group B & \multirow{2}{*}{ P Value } \\
\cline { 2 - 3 } & Mean \pm SD & Mean \pm SD & \\
\hline AGE (Years) & $54.00 \pm 6.25$ & $52.34 \pm 7.57$ & 0.224 \\
BMI(Weight/ $\mathbf{K g}^{2}$ ) & $24.90 \pm 3.95$ & $24.86 \pm 4.76$ & 0.976 \\
\hline GENDER (\%) & $\mathrm{M}: 48.3 \%$ & $\mathrm{M}: 51.7 \%$ & \\
& $\mathrm{~F}: 51.7 \%$ & $\mathrm{~F}: 48.3 \%$ & - \\
\hline PRE-VAS(Cm) & $5.48 \pm 1.24$ & $5.63 \pm 0.97$ & 0.615 \\
\hline PRE SPADI (\%) & $54.00 \pm 13.99$ & $53.086 \pm 13.34$ & 0.798 \\
\hline PRE-FLEXION & $117.88 \pm 32.86$ & $113.33 \pm 24.61$ & 0.674 \\
\hline PRE-EXTENSION & $34.23 \pm 11.46$ & $30.93 \pm 10.09$ & 0.196 \\
\hline PRE-ABDUCTION & $79.42 \pm 23.25$ & $68.52 \pm 22.52$ & 0.157 \\
\hline PRE ER & $32.88 \pm 16.80$ & $29.63 \pm 17.26$ & 0.667 \\
\hline PRE IR & $30.38 \pm 17.25$ & $31.67 \pm 15.44$ & 0.581 \\
\hline
\end{tabular}

Table 2: Intragroup comparison of vas, SPADI and rom by using paired t-test.

\begin{tabular}{|c|c|c|c|c|c|c|}
\hline \multirow{3}{*}{ Variable } & \multicolumn{2}{|c|}{ Group A } & \multirow{3}{*}{ P value } & \multicolumn{2}{|c|}{ Group B } & \multirow{3}{*}{ P value } \\
\hline & Pre intervention & Post intervention & & Pre intervention & Post intervention & \\
\hline & (Mean \pm SD) & (Mean \pm SD) & & (Mean \pm SD) & (Mean \pm SD) & \\
\hline VAS & $5.48 \pm 1.28$ & $3.16 \pm 1.05$ & 0 & $5.61 \pm 1.01$ & $4.06 \pm 1.07$ & 0 \\
\hline SPADI & $53.62 \pm 14.41$ & $22.67 \pm 9.96$ & 0 & $52.43 \pm 13.92$ & $31.14 \pm 12.00$ & 0 \\
\hline Flexion & $117.22 \pm 32.41$ & $135.37 \pm 32.55$ & 0 & $113.85 \pm 24.95$ & $127.12 \pm 23.58$ & 0 \\
\hline Extension & $34.44 \pm 11.29$ & $43.89 \pm 9.02$ & 0 & $30.58 \pm 10.15$ & $39.04 \pm 9.59$ & 0 \\
\hline Abduction & $78.33 \pm 23.49$ & $110.56 \pm 17.61$ & 0 & $69.23 \pm 22.65$ & $93.46 \pm 22.44$ & 0 \\
\hline External Rotation & $32.22 \pm 16.83$ & $53.70 \pm 17.40$ & 0 & $30.19 \pm 17.34$ & $41.92 \pm 17.78$ & 0 \\
\hline Internal Rotation & $29.81 \pm 17.18$ & $43.70 \pm 15.84$ & 0 & $32.31 \pm 15.37$ & $43.65 \pm 15.20$ & 0 \\
\hline
\end{tabular}

Table 1: Patient's Baseline characteristics compared for homogeneity by unpaired t test.

\begin{tabular}{lcccc}
\hline \multirow{2}{*}{ Variable } & Group A & Group B & \\
\cline { 2 - 3 } & Pre-post diff & Pre-post diff & \multirow{2}{*}{ P value } \\
\cline { 2 - 3 } & Mean \pm SD & Mean \pm SD & \\
\hline VAS & $-2.315 \pm 0.99$ & $-1.55 \pm 0.72$ & 0.002 \\
\hline SPADI & $-30.95 \pm 8.71$ & $-21.28 \pm 7.43$ & 0 \\
\hline Flexion & $18.15 \pm 9.31$ & $13.27 \pm 6.92$ & 0.036 \\
Extension & $9.44 \pm 4.23$ & $8.46 \pm 3.08$ & 0.341 \\
Abduction & $32.22 \pm 11.37$ & $24.23 \pm 13.54$ & 0.024 \\
External Rotation & $21.48 \pm 11.73$ & $11.73 \pm 8.59$ & 0.002 \\
\hline Internal Rotation & $13.89 \pm 8.12$ & $11.35 \pm 6.29$ & 0.209 \\
\hline
\end{tabular}

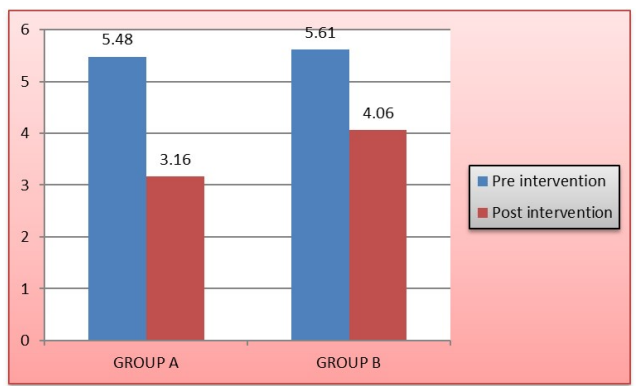

Graph 1: Intra and inter group comparison of VAS

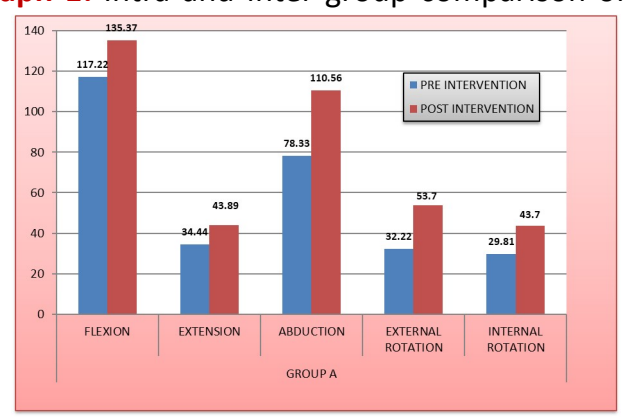

Graph 3: Intragroup comparison of ROM (Group A)

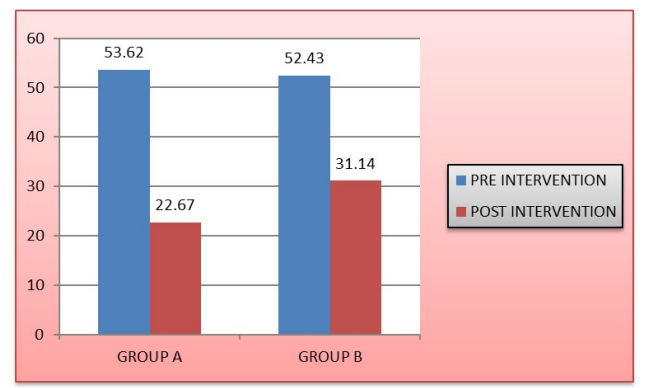

Graph 2: Intra and inter group comparison of SPADI

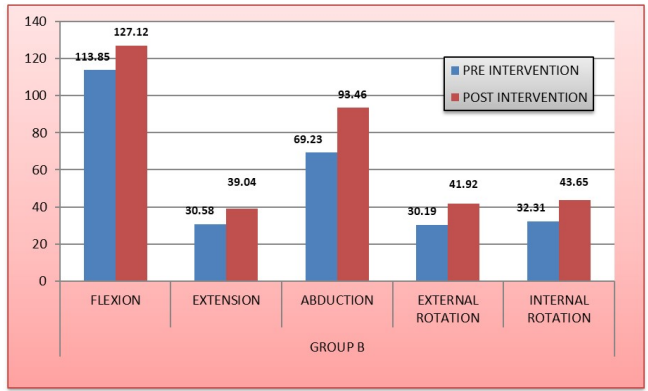

Graph 4: Intragroup comparison of ROM (Group B) 
Result of present study showed that in 4-week intervention both the groups showed statistically significant improvement in VAS score, SPADI scores and ROM post intervention compared to pre intervention measures. When compared between group Spencer MET group showed more effectiveness in reducing pain, disability and increasing external rotation, abduction and flexion ROM than Maitland Mobilisation group. Improvement in internal rotation and extension ROM showed no significant difference between both the groups.

In Group A, pain was reduced after intervention, the possible mechanism include neurological and tissue factors, such as stimulation of low threshold mechanoreceptors on centrally mediated pain inhibitory mechanism and on neuronal populations in the dorsal horn with possible gating effect. Low threshold mechanoreceptors from the joints and muscles project to the peri-aqueductal grey in the midbrain region. During isometric contraction, activation of muscle and joint mechanoreceptors occur. This leads to sympatho-exitation evoked by somatic efferent's and localized activation of PAG that plays a role in descending modulation of pain. Nociceptive inhibition then occurs at the dorsal horn of the spinal cord, as simultaneous gating takes place of nociceptive impulses in dorsal horn, due to mechanoreceptor stimulation [18].

In group B, pain was also reduced after intervention; A Maitland mobilization oscillatory glide reduces pain by stimulating natural pain-relieving substances like endorphins. Oscillatory movements stimulate mechanoreceptors associated with the myelinated alpha beta and alpha delta fibres. The impulses stimulated by mobilization there by block the pain impulse and break the pain cycle by activating the pain gate, which consequently lessened suffering in daily activities, pain with specific tasks, and difficulty in moving arm and lifting actions. When patient's pain decreased, it revealed a reduction in SPADI scores. Mobilization provokes golgi tendon organ. at the end of the joint mobilization and causes reflex inhibition of the muscle which was concluded by lundberg A et al in 1978 [20]. Both the groups had received hot pack. The probable mechanism for pain relief is that the superficial heating effect of heat therapy increases the temperature of localized tissue, so vascular dilatation is promoted, and the pain threshold elevated. Such vascular improvement also accelerates the process of inflammation by increasing nutrition and oxygen supply, and by removing metabolites and waste products. This leads to a decrease in pain and swelling.

Both the groups had received Conventional Exercises. The probable mechanism for pain relief is that Exercises within the pain free range of motion stimulates mechanoreceptors and decreases pain. Exercises within pain free range also move the synovial fluid, thus decrease inflammation and decreased pain [13]. Patients were not told to change their usual medication, which may have helped in reducing pain. However, $90 \%$ of the patients stopped their medication during treatment duration for both the groups.

For Group A the mechanism behind increase in ROM by MET is that muscle contraction against equal counterforce triggers the Golgi tendon organ. The afferent nerve impulse from the Golgi tendon organ enters the dorsal root of the spinal cord and meets with an inhibitory motor neurone. This stops the discharge of the efferent motor neurones impulse and therefore prevents further contraction, the muscle tone decreases, which in turn results in the agonist relaxing and lengthening, so there is increase in the ROM. The finding of the present study is similar as found by Gupta S, Jaiswal P (2008) [21]. Suggesting that Post isometric relaxation is more effective in decreasing pain and disability and improving cervical range of motion as compared to isometric exercises over a period of three weeks in patients having non-specific neck pain.

For Group B the mechanism increase in ROM by $\mathrm{MM}$ may be due to mechanical force during mobilization leading to breaking up of adhesions, realigning biological changes in synovial fluid and their enhanced exchange [22]. MM improves normal extensibility of 
capsule, restore joint play and stretches the tightened soft tissues and periarticular structures to induce beneficial effects [23]. These techniques are also thought to increase the proprioceptive and kinaesthetic sensation in the joint thus subjects can do the activities in newly gained range of motion [24]. $\mathrm{MM}$ is believed to increase or maintain joint mobility by inducing rheological changes in synovial fluid, better exchange between synovial fluid and cartilage matrix, and increased synovial fluid turnover [25].

Improvement in internal rotation and extension ROM showed no significant difference between both the groups. The reason for this finding has been explained below. According to position of SPENCER MET stage 7, which was given for increasing internal rotation ROM requires extreme internal rotation range as starting position. So, in initial treatment period because of pain patients in these groups were not able to assume the starting position. Hence this stage was introduced late during the intervention. The affection of extension was less pre intervention in both the groups. So, the difference of post intervention measures compared to pre intervention was less in both the groups. That can be possible reason for no significant difference between groups.

Findings of present study are like that of Edrish Saifee Contractor, et al. [26] Who studied Effect of Spencer Muscle Energy Technique on pain and functional disability in cases of adhesive capsulitis of shoulder joint over conventional physiotherapy. However, no previous studies compared SPENCER MET with $\mathrm{MM}$ in frozen shoulder patients.

Limitations of the study are long term follow up of the patients was not done after completion of the intervention duration; hence long term benefits of intervention are unknown. Absence of control group cannot justify natural recovery during intervention period in both the groups.

\section{CONCLUSION}

This study concludes that both the techniques used in the present study i.e. Spencer Muscle Energy Technique and Maitland Mobilization are effective for improving pain, reducing disability and increasing ROM, when given along with conventional exercises. However, SPENCER MET is the more effective to improving pain, reducing disability and increasing ROM compared to Maitland Mobilization in patients with frozen shoulder.

\section{ABBREVIATIONS}

MET - Muscle Energy Technique

ROM - Range of Motion

VAS - Visual Analouge Scale

SPADI - Shoulder Pain and Disability Index

SPSS - Statistical Package for the Social Sciences

FS - Frozen Shoulder

PIR - Post Isometric Relaxation

RI - Reciprocal Inhibition

MM - Maitland mobilization

OPD - Out Patient Department

BMI - Body Mass Index

SD - Standard Deviation

ER - External Rotation

IR - Internal Rotation

\section{ACKNOWLEDGEMENTS}

We are thankful to God, Principal of SPB Physiotherapy College and all the participants of the study for their support and co-operations.

\section{Conflicts of interest: None}

\section{REFERENCES}

[1]. Codman EA. The Shoulder.Boston, Massachusetts: Thomas Todd Company; 1934.

[2]. Andrew S. Neviaser, Jo A. Hannafin. Adhesive Capsulitis: A Review of Current Treatment. The American Journal of Sports Medicine, 2010;38(11):2346-2356.

[3]. Usman Iqbal Janjua, Shaukat Ali. Physical Therapy \& Maitland's Manual Joint Mobilization Techniques (GRADE II \& III) are effective to manage the stage I Adhesive Capsulitis. Interdisciplinary Journal of Contemporary Research in Business, 2011;3(8):243-248

[4]. James P. Tasto, David W. Elias. Adhesive Capsulitis. Sports Med Arthrose Rev., 2007;15(4):216-221.

[5]. Duplay ES. De la periarthrite scapulohumerale et des raideurs de l'epaule qui en son la consequence. Arch Gen Med., 1872;20:513-542.

[6]. Adkitte R, Rane SG, Yeole U, Nandi B, Gawali P. Effect of muscle energy technique on flexibility of hamstring muscle in Indian national football players. Saudi J Sports Med 2016;16:28-31.

[7]. Neviaser JS, Washington DC. Adhesive capsulitis of the shoulder. J Bone Joint Surg Am., $1945 ; 17(2): 211-222$. 
[8]. John Gibbon. Introduction to muscle energy technique. International Therapist. July 2011;97:26-28.

[9]. DA Patriquin. The evolution of osteopathic manipulative technique: The spencer technique. The Journal of the American Osteopathic Association 1992; 62(9): 1134.

[10]. Robert C. Ward. Foundations for Osteopathic Medicine. 2nd ed. Lippincott Williams \& Wilkins; 2003.

[11]. ES Contractor, DS Agnihotri, RM Patel - Effect of Spencer Muscle Energy Technique on pain and functional disability in cases of adhesive capsulitis of shoulder joint -iaimjournal.com 2016; 3(8):126131.

[12]. Maitland GD. Treatment of the glenohumeral joint by passive movement. Physiotherapy, 1983;69: 3-7.

[13]. Abhay Kumar, Suraj Kumar, Anoop Agarwal, Ratnesh Kumar, and Pooja Ghosh Das. Effectiveness of Maitland Techniques in Idiopathic Shoulder Adhesive Capsulitis. 2012;10:5402.

[14]. Leighann Litchr Kelly, Sharon A. Martino, Joan E. Broderick and Arthur A. Stone. A systemic review of measures used to assess chronic musculoskeletal pain in clinical and randomized controlled clinical trials. Journal of pain 2007;8(12):906-913.

[15].Richard L Gajdosik and Richard W Bohannon. Review of goniometry emphasizing reliability and validity. Journal of the American Physical Therapy Association 1987;67(12):1867-72.

[16]. Riddle DL, Rothstein JM, Lamb RL, Goniometric reliability in a clinical setting. Shoulder measurements. Physical Therapy 1987;67(5):668-73.

[17]. Breckenridge JD, Mc Auley JH. Shoulder pain and disability index (SPADI). Journal of Physiotherapy 2011;57(3):197.

[18]. Leon Chaitow. Muscle Energy Techniques. $3^{\text {rd }}$ ed. Churchill Livingstone Elsevier; 2006.
[19]. Eileen L. DiGiovanna, Stanley Schiowitz, Dennis J. Dowling. An Osteopathic Approach to Diagnosis and Treatment. $3^{\text {rd }}$ ed. Lippincott Williams \& Wilkins; 2005.

[20]. Lundberg A, malmgren: Role of joint afferents in motor control exemplified by effects on reflex pathways from 1b afferents. 1978;284:327-343.

[21]. Gupta S, Jaiswal, P, Chhabra D. A Comparative Study between Postisometric Relaxation and Isometric Exercises in Non-Specific Neck Pain. Journal of Exercises Science and Physiotherapy, 2008;4(2): 88-94.

[22].Donateli R, Wooden JM. Orthopedic physical therapy, Churchill Leving stone, New York, NY, USA, $2^{\text {nd }}$ edition (1994).

[23]. Vermeulen, H.M., Obermann, W.R. and Burger.B.J. End-range mobilization techniques in adhesive capsulitis of the shoulder joint: a multiple -subject case report. Physical therapy 2000;80:12041213.

[24]. Kumar A, kumar S, et.,al.Effectiveness of maitland technique in idiopathic shoulder Scholarly Reasearch Network ISRN rehabilitation.2012;1:8.

[25]. Zaky AL. End range mobilization (ERM) versus mobilization with movement (MWM) in treatment of adhesive capsulitis. Bulletin of faculty of physical therapy cairo university.2012;17(2):47-53.

[26]. Edrish saifee contractore, et al. Effect of Spencer Muscle Energy Technique on pain and functional disability in cases of adhesive capsulitis of shoulder joint. International Archives of Integrated Medicine, 2016;3(8).

How to cite this article:

Raksha R. Jivani, Dharti N Hingarajia. Effect of Spencer Muscle Energy Technique Versus Maitland's Mobilization Technique on Pain, ROM and Disability in Patients with Frozen Shoulder: A Comparative Study. Int J Physiother Res 2021;9(4):3928-3936. DOI: 10.16965/ijpr.2021.148 